\title{
Congenital Insensitivity to Pain With Anhidrosis (CIPA): Novel Mutations of the TRKA (NTRK1) Gene, a Putative Uniparental Disomy, and a Linkage of the Mutant TRKA and PKLR Genes in a Family With CIPA and Pyruvate Kinase Deficiency
}

\author{
Yasuhiro Indo, ${ }^{1 *}$ Sek Mardy, ${ }^{1}$ Yuichi Miura, ${ }^{1}$ Allie Moosa, ${ }^{2}$ Essam A.R. Ismail, ${ }^{3}$ Ennio Toscano, ${ }^{4}$ \\ Generoso Andria, ${ }^{4}$ Vito Pavone, ${ }^{5}$ Deborah L. Brown, ${ }^{6}$ Alice Brooks, ${ }^{7}$ Fumio Endo, ${ }^{1}$ and \\ Ichiro Matsuda ${ }^{1}$ \\ ${ }^{1}$ Department of Pediatrics, Kumamoto University School of Medicine, Kumamoto, Japan \\ ${ }^{2}$ Department of Pediatrics, Faculty of Medicine, Kuwait University, Safat, Kuwait \\ ${ }^{3}$ Department of Pediatrics, Farwaniya Hospital, Salmiya, Kuwait \\ ${ }^{4}$ Department of Pediatrics, Federico II University, Naples, Italy \\ ${ }^{5}$ Orthopedic Clinic, University of Catania, Italy \\ ${ }^{6}$ Hematology-Oncology, Children's Memorial Hospital Chicago, Chicago, Illinois \\ ${ }^{7}$ Department of Clinical Genetics, Erasmus University Rotterdam, Rotterdam, The Netherlands
}

Communicated by Richard G.H. Cotton

\begin{abstract}
Congenital insensitivity to pain with anhidrosis is an autosomal recessive hereditary disorder characterized by recurrent episodic fever, anhidrosis (inability to sweat), absence of reaction to noxious stimuli, self-mutilating behavior, and mental retardation. The human TRKA gene (NTRK1), located on chromosome 1q21-q22 encodes the receptor tyrosine kinase for nerve growth factor. We reported that TRKA is the gene responsible for CIPA and we developed a comprehensive strategy to screen for TRKA mutations and polymorphisms, as based on the gene's structure and organization. Here we report eight novel mutations detected as either a homozygous or heterozygous state in nine CIPA families from five countries. Mendelian inheritance of the mutations was confirmed in seven families for which samples from either parent were available. However, nonmendelian inheritance seems likely for the family when only samples from the mother and siblings, (but not from the father) were available. A paternal uniparental disomy for chromosome 1 is likely to be the cause of reduction to homozygosity of the TRKA gene mutation in this family. Interestingly, a Hispanic patient from the USA has two autosomal genetic disorders, CIPA and pyruvate kinase deficiency, whose genetic loci are both mapped to a closely linked chromosomal region. A splice mutation and a missense mutation were detected in the TRKA and PKLR genes from the homozygous proband, respectively. Thus, concomitant occurrence of two disorders is ascribed to a combination of two separate mutant genes, not a contiguous gene syndrome. This finding suggests a mechanism responsible for two autosomal genetic disorders in one patient. All these data further support findings that TRKA defects can cause CIPA in various ethnic groups. This will aid in diagnosis and genetic counseling of this painless but severe genetic disorder. Hum Mutat 18:308-318, 2001. (ㅇ) 2001 Wiley-Liss, Inc.
\end{abstract}

Received 20 February 2001; accepted revised manuscript 31 May 2001.

*Correspondence to: Dr. Yasuhiro Indo, Department of Pediatrics, Kumamoto University School of Medicine, Honjo 1-1-1, Kumamoto 860-8556, Japan.

E-mail: yindo@kaiju.medic.kumamoto-u.ac.jp

Contract grant sponsors: Ministry of Education, Culture, Sports,
Science and Technology of Japan; Ministry of Health, Labour and Welfare of Japan.

Current address for Sek Mardy: Experimental Retrovirology Section, National Cancer Institute, National Institutes of Health, Bethesda, Maryland.

Current address for Ichiro Matsuda: Ezuko Institution for Developmental Disabilities, Kumamoto, Japan. 
KEY WORDS: congenital insensitivity to pain with anhidrosis; CIPA; TRKA; NTRK1; nerve growth factor; NGF; receptor tyrosine kinase; PKLR; hereditary sensory and autonomic neuropathy type IV; HSAN-IV; pyruvate kinase deficiency

DATABASES:

NTRK1 - OMIM: 191315, 256800 (CIPA); GDB: 127897; GenBank: AB019480-AB019488, M23102; HGMD: NTRK1

PKLR - OMIM: 266200; GDB: 120294; HGMD: PKLR

\section{INTRODUCTION}

Congenital insensitivity to pain with anhidrosis (CIPA; MIM\# 256800) is an autosomal recessive disorder characterized by recurrent episodic fever depending on high environmental temperature, anhidrosis (inability to sweat), absence of reaction to noxious stimuli, self-mutilating behavior, and mental retardation [Dyck, 1984; McKusick, 1994; Swanson, 1963]. CIPA is also classified as hereditary sensory and autonomic neuropathy type IV [Dyck, 1984]. Various neurotrophic factors (neurotrophin) support cell survival, growth, and differentiation in nervous systems. Nerve growth factor (NGF), the first neurotrophic factor to be identified, supports the survival of sympathetic ganglion neurons and nociceptive sensory neurons in dorsal root ganglia, derived from the neural crest, and ascending cholinergic neurons of the basal forebrain [Levi-Montalcini, 1987; Thoenen and Barde, 1980]. TRKA is a receptor tyrosine kinase that is phosphorylated in response to NGF [Kaplan et al., 1991; Klein et al., 1991].

We identified the genetic basis for CIPA when we detected loss-of-function mutations in the TRKA (also NTRK1; MIM\# 191315) gene derived from affected patients [Indo et al., 1996]. CIPA is the first human genetic disorder implicated in the neurotrophin signal transduction system. The anomalous pain and temperature sensation and anhidrosis in CIPA are probably because of the absence of afferent neurons activated by tissue-damaging stimuli and a loss of sympathetic innervation of eccrine sweat glands, respectively. Human TRKA (NTRK1) gene maps to chromosome 1q21-q22 [Weier et al., 1995]. Recently, we developed a strategy to screen for TRKA mutations, on the basis of the structure and organization of the gene [Indo et al., 1997; Mardy et al., 1999] and we characterized eight intragenic polymorphic sites [Miura et al., 2000b]. Other investigators have also detected and reported mutations in the TRKA from CIPA patients [Greco et al., 1999; Shatzky et al., 2000; Yotsumoto et al., 1999].
We have also identified a complete paternal isodisomy for chromosome 1 as the cause of reduction to homozygosity of the TRKA gene mutation, leading to CIPA [Miura et al., 2000a]. Recently, we examined effects of TRKA missense mutations on autophosphorylation of the receptor tyrosine kinase for NGF [Mardy et al., 2001]. These studies led to the genetic diagnosis of CIPA, which was previously established on the basis of clinical findings, pharmacological tests, and peripheral nerve biopsy.

We report here clinical profiles and eight novel TRKA mutations as homozygous or compound heterozygous in nine families with CIPA from Kuwait, Italy, the United States, The Netherlands, and Japan. We also describe a putative uniparental disomy as the cause of reduction to homozygosity of the TRKA gene mutation in a family and a linkage of two autosomal recessive genetic disorders, CIPA and pyruvate kinase deficiency, in another family.

\section{Patients}

\section{MATERIALS AND METHODS}

The nine affected families with CIPA were three each from Kuwait (KI-109, KI-110, and KI-111) and Italy (KI-112, KI-113, and KI-114); and one each from the United States (KI-115), The Netherlands (KI-116), and Japan (KI-25). Blood was collected from patients with CIPA and from family members referred to the investigators. Informed consent was obtained from all these tested subjects.

Family KI-109. The proband is a 3-month-old boy born to consanguineous parents. He has three siblings who are unaffected. He first presented at the age of $24 \mathrm{hr}$ with fever that persisted. He did not respond to painful stimuli such as blood sampling. Extensive investigations revealed no infectious cause for the fever. The parents reported that he was never seen sweating. This family was referred for the genetic diagnosis of CIPA.

Family KI-110. The proband is a 2-year-old boy with unexplained fevers, febrile convulsions, 
and mutilation of his tongue for which he had all teeth extracted. He did not respond to painful stimuli such as blood sampling. Extensive investigations revealed no infectious cause for the fever. He is hypotonic, anhidrotic, and delayed in development. This family was also referred for the genetic diagnosis of CIPA.

Family KI-111. The patient is a 9-year-old girl born to consanguineous parents. She has two unaffected younger siblings (a brother and a sister). During infancy, she often had unexplained fever especially in the hot weather. She shows no response to noxious stimuli and no sweating under hot environmental conditions. She has demonstrated self-mutilating behavior such as avulsion of teeth and biting of fingertips. Her right leg is amputated below the knee and several toes on her left foot are missing.

Family KI-112. The patient, a 13-year-old boy, presents a complete phenotype of CIPA, including recurrent episodes of unexplained fever, anhidrosis, absence of reaction to noxious stimuli, self-mutilating behavior, and mental retardation. Parents denied consanguinity.

Family KI-113. The patient was a 22-year-old man, the first child of healthy consanguineous parents (first cousins), who was previously reported as "two brothers with a variant of hereditary sensory neuropathy" [Pavone et al., 1992]. His clinical phenotype was described as a variant form of CIPA because a sural nerve biopsy showed neuropathological findings consistent with this disorder but with normal sweating function and absence of recurrent fever. However, re-examination with pilocarpine iontophoresis showed that this patent was indeed anhidrotic. He died at age 25 years due to renal dysfunction and spine fractures. His younger brother with the same phenotype died at age 15 years due to an infection-related disorder. Data on their six-year-old younger sister were not previously reported but she presents a CIPA phenotype.

Family KI-114. The patient, an 18-year-old girl, presents a mild phenotype with insensitivity to pain, hypohidrosis, severe bone involvement, and no apparent mental retardation. Parents are not consanguineous.

Family KI-115. The patient is a five-year-old Hispanic boy whose parents were born in a small town in Mexico, but are not known to be related. He began to exhibit self-mutilating behavior in infancy after his teeth erupted. He required three surgeries for tongue reconstruction and eventually had all of his primary teeth extracted. He has also been biting and pulling out his fingernails. By age four, he had fractures in metatarsals of both feet, without any known trauma and no signs of pain. He developed deep ulcerations after casts were put on but appeared to have no pain. He has had recurrent hyperpyrexia with fevers as high as $41^{\circ} \mathrm{C}$ and experienced two episodes of febrile seizures. He frequently has a fever in warm weather or when he is overdressed but no observed diaphoresis. His growth is poor, with height and weight consistently below the 5 th percentile, and he has developmental delay as well as hyperkinetic behavior. Examination of nerve conduction velocities and a sural nerve biopsy confirmed the diagnosis of CIPA. In addition to neurological findings, he has had since birth a moderately severe hemolytic anemia. Initially, he received sporadic transfusions when his hemoglobin dropped as low as $5 \mathrm{~g} / \mathrm{dl}$, usually associated with an intercurrent illness. At age three years, pyruvate kinase deficiency was diagnosed based on an enzyme assay by Dr. E. Beutler's laboratory at Scripps Research Institute. Both his parents had enzyme levels consistent with heterozygosity. The father has a mild hemolytic anemia and the mother is clinically asymptomatic. At age 51/2 years a splenectomy was done, with minimal benefits, and repetitive transfusions with iron-chelation therapy were given to the boy.

Family KI-116. The patient is a 7-year-old boy. His parents are of Dutch ancestry and consanguinity is denied. Postnatally hypothermia was present, probably due to anomalous regulation of his body temperature. At age six months he developed unexplained febrile episodes in the summer months. He did not want to walk even at the age of five years. His ankles were swollen but seemed to be painless. Radiological studies were suggestive of arthritis. He presents anhidrosis and has hyperkeratosis of the palms and soles. His response to painful stimuli is nil. Intradermal injection of histamine provoked no axonal reflex. He was developmentally retarded and a formal assessment revealed that his IQ was 70 .

Family KI-25. The patient is a 2-year-old girl born normally at term after an uneventful pregnancy to non-consanguineous healthy Japanese parents. At age four months she presented with 
an oral ulcer that seemed to be painless. She also had febrile episodes but extensive investigations revealed no cause. She did not cry during blood sampling. She did not sweat and her skin was dry. Skin biopsy indicated the presence of eccrine sweat glands but their innervation was absent. Conduction velocity of peripheral nerves was within a normal range. Sural nerve biopsy revealed a decrease of unmyelinated and small myelinated nerves. She was hypotonic and developmentally retarded. She repeated self-mutilating behaviors such as biting her tongue.

\section{Genetic Analysis}

Numbering system for amino acids and nucleotides. Mutations are designated according conventional nomenclature [Antonarakis, 1998], using the TRKA cDNA sequence and amino acid numbering [Martin-Zanca et al., 1989], and the structure and organization of the human TRKA gene [Indo et al., 1997].

TRKA amplifications and DNA sequencing. TRKA exons were amplified from genomic DNA using specific primers derived from the $5^{\prime}$ and $3^{\prime}$ intronic or exonic sequences, and AmpliTaq Gold (Applied Biosystems, Tokyo, Japan) or the Expand Long PCR system enzyme (Roche Molecular Biochemicals, Mannheim, Germany). The corresponding polymerase chain reaction (PCR) products were sequenced directly. The primers and sequencing of PCR products have been described elsewhere [Mardy et al., 1999]. Sequencing of the PCR products was done using dRhodamine Terminator Cycle Sequencing Kits (Applied Biosystems, Tokyo, Japan). Sequences were resolved and analyzed on an ABI PRISM 310 Genetic Analyzer (Applied Biosystems, Tokyo, Japan).

Restriction digestion analysis. For analysis of Gln176Ter mutation from KI-109 and KI-110 families, genomic DNA was amplified and the following set of primers was used. Forward primer: 5'-CTCCCTCTTATCCCCTGTGA-3'; reverse primer: 5'-TTTCAGTGCTTGCTCAGTGCC-3'). PCR products were digested with a restriction enzyme PstI and electrophoresed onto an agarose gel.

\section{Haplotype and Allelotype Analyses}

In family KI-111, haplotypes of the proband and her mother were characterized using eight single nucleotide polymorphic markers and one microsatellite marker (D1S506) within the TRKA locus, as described elsewhere [Cargill et al., 1999; Miura et al., 2000b]. Microsatellite markers outside the TRKA locus spanning the entire chromosome 1 were characterized. Each marker was amplified from genomic DNA by AmpliTaq Gold and primer pairs of the Linkage Mapping Set Version 2 (Applied Biosystems), according to the manufacturer's protocol. PCR products were resolved on an ABI PRISM 310 Genetic Analyzer, and the results were analyzed using ABI PRISM GeneScan and Genotyper software (Applied Biosystems).

\section{RESULTS \\ Mutation Analysis of TRKA Gene in CIPA Families}

Nine unrelated CIPA families were screened for mutations in the TRKA gene. All 17 exons of the TRKA gene, including their flanking intronic sequences, were amplified from the genomic DNA of each patient with CIPA. All exons and intron-exon boundaries were sequenced. Mutations detected by PCR methods were confirmed on a second PCR product and were not detected in 100 normal chromosomes [Mardy et al., 1999; Miura et al., 2000b].

In family KI-109, we found a $\mathrm{C}$ to $\mathrm{T}$ transition at nucleotide 610 in exon 5 , which changes a Gln to a termination codon at amino acid 176 (Gln176Ter) in the proband (Table 1 and Fig. $1)$. Parents and three siblings were also sequenced. The substitution destroys a restriction site for PstI. Digestion analysis with this enzyme also demonstrated that the proband and parents are homozygous and heterozygous, respectively. Three unaffected siblings were heterozygous for the substitution.

In family KI-110, we also detected the same substitution as described for KI-109 (Table 1 and Fig. 1). Parents were also sequenced. The proband and parents were homozygous and heterozygous for this substitution, respectively. These two families, KI-109 and KI-110, are not apparently related although they are from the same countries.

In family KI-111, direct sequencing of samples from the patient revealed a homozygous eight base pair insertion (GGGACATC) between nucleotides 1596 and 1597 in exon 13, which causes a frameshift and a truncation after amino acid Val 505 (Table 1 and Fig. 1). Mother and 
TABLE 1. Mutation Analysis of CIPA Families

\begin{tabular}{|c|c|c|c|c|}
\hline Family & Status & $\begin{array}{c}\text { Nucleotide change } \\
\text { (location) }\end{array}$ & $\begin{array}{c}\text { Mutation type \& } \\
\text { (predicted consequence) }\end{array}$ & $\begin{array}{l}\text { Country } \\
\text { of origin }\end{array}$ \\
\hline KI-109 & Homozygote & $\begin{array}{l}\text { c.610C }>\mathrm{T} \\
\text { (exon } 5)\end{array}$ & $\begin{array}{l}\text { Nonsense } \\
\text { (Gln176Ter) }\end{array}$ & Kuwait \\
\hline KI-110 & Homozygote & $\begin{array}{l}\text { c.610C }>\mathrm{T} \\
(\text { exon } 5)\end{array}$ & $\begin{array}{l}\text { Nonsense } \\
\text { (Gln176Ter) }\end{array}$ & Kuwait \\
\hline $\mathrm{KI}-111^{\mathrm{a}}$ & Homozygote & $\begin{array}{l}\text { c.1596 } 1597 \text { insGGGA } \\
\text { CATC } \\
\text { (exon 13) }\end{array}$ & $\begin{array}{l}\text { Frameshift } \\
\text { (truncation after Val505) }\end{array}$ & Kuwait \\
\hline $\mathrm{KI}-112^{\mathrm{b}}$ & Homozygote & $\begin{array}{l}\text { c.475-476delTC } \\
\text { (exon 4) }\end{array}$ & $\begin{array}{l}\text { Frameshift } \\
\text { (truncation after Ser131) }\end{array}$ & Italy \\
\hline KI113 & Homozygote & $\begin{array}{l}\text { c.1588-1589delCG } \\
\text { (exon 13) }\end{array}$ & $\begin{array}{l}\text { Frameshift } \\
\text { (truncation after Arg502) }\end{array}$ & Italy \\
\hline KI-114 & $\begin{array}{l}\text { Compound } \\
\text { heterozygote }\end{array}$ & $\begin{array}{l}\text { c. } 2337 \mathrm{C}>\mathrm{G}(\text { exon } 17) \\
\text { c. } 2347 \mathrm{C}>\mathrm{T}(\text { exon } 17)\end{array}$ & $\begin{array}{l}\text { Nonsense (Tyr751Ter) } \\
\text { Missense (Arg 755Trp) }\end{array}$ & Italy \\
\hline $\mathrm{KI}-115^{\mathrm{c}}$ & Homozygote & IVS9-1G > A (intron 9) & $\begin{array}{l}\text { Splice site } \\
\text { (aberrant splicing of exon 10) }\end{array}$ & $\begin{array}{l}\text { USA } \\
\text { (Mexican) }\end{array}$ \\
\hline KI-116 & Homozygote & $\begin{array}{l}\text { c.2347C }>\mathrm{T} \\
\text { (exon } 17)\end{array}$ & $\begin{array}{l}\text { Missense } \\
\text { (Arg 755Trp) }\end{array}$ & Netherlands \\
\hline KI-25 & $\begin{array}{l}\text { Compound } \\
\text { heterozygote }\end{array}$ & $\begin{array}{l}\text { IVS6+1G }>C \text { (intron 6) } \\
\text { c. } 2210 \mathrm{~T}>\mathrm{C}(\text { exon } 16)\end{array}$ & $\begin{array}{l}\text { Splicing (aberrant splicing of exon 6) } \\
\text { Missense (Val709Ala) }\end{array}$ & Japan \\
\hline
\end{tabular}

Positions of nucleotide change are from the transcription start site, as described [Martin-Zanca et al., 1989]. The ATG initiation codon is located at nucleotide position c.85. Structure and organization of human TRKA have been documented [Indo et al., 1997].

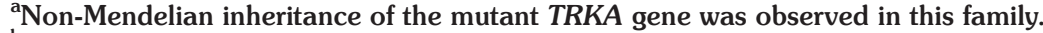

${ }^{\mathrm{b}}$ This mutation was previously reported as one allele of a Japanese patient [Miura et al., 2000b].

${ }^{\mathrm{c}}$ The linkage of the mutant TRKA and PKLR genes observed in this family is responsible for CIPA and pyruvate kinase deficiency, respectively.

two siblings were also sequenced. However, we did not detect mutation in the DNA samples from the mother and two siblings. DNA from the father was not available. We confirmed this sequence analysis when we used DNA from fresh blood samples. To define the mode of inheritance in this family, we characterized haplotypes of the proband and her mother, using eight single nucleotide polymorphic markers and one microsatellite marker (D1S506) within the TRKA locus, as described elsewhere [Cargill et al., 1999; Miura et al., 2000a; Miura et al., 2000b]. Polymorphism analysis of the TRKA locus revealed that the patient was homozygous (Fig. 1: lower part). Analyses of the single nucleotide polymorphism (SNP) site in intron 5 and the D1S506 in intron 12 revealed that the patient and her mother were homozygous and heterozygous, respectively. Her two siblings were also heterozygous for the D1S506. Thus, we excluded the possibility that the mother had a large deletion in the TRKA gene. Further analysis of the SNP site in exon 14 (c.1794 C/T) revealed that the patient and her mother were homozygous $\mathrm{C}$ and $\mathrm{T}$, respectively (Fig. 1: lower part). These analyses strongly indicate the non-Mendelian inheritance of the TRKA locus haplotype in this family.
Two bases TC at nucleotides 475-476 were deleted in exon 4 in a male patient with CIPA of family KI-112 (Table 1 and Fig. 1). The deletion causes a frameshift and premature termination codon after amino acid Ser 131. Parents were also sequenced. The patient and parents were homozygous and heterozygous, respectively. Thus, the deletion is apparently the cause of CIPA in this family. The same mutation was previously reported in a Japanese family [Miura et al., 2000b]. We determined a haplotype of the proband in Italian family KI-112. The proband was homozygous for nine polymorphic sites of the TRKA locus that we examined. This strongly suggests that parents share the same TRKA mutant allele linked to the same haplotype, although they denied consanguinity.

In family KI-113, two bases CG at nucleotides 1588-1589 were deleted in exon 13 of the two siblings, a male and a female (Table 1 and Fig. 1). Parents were also sequenced. DNA sample from the younger boy was not available for analysis. The deletion causes a frameshift and premature termination codon after amino acid Arg 502. The patients and parents were homozygous and heterozygous, respectively. Thus, this deletion is apparently the cause of CIPA in this family.

In family KI-114, the proband was a com- 


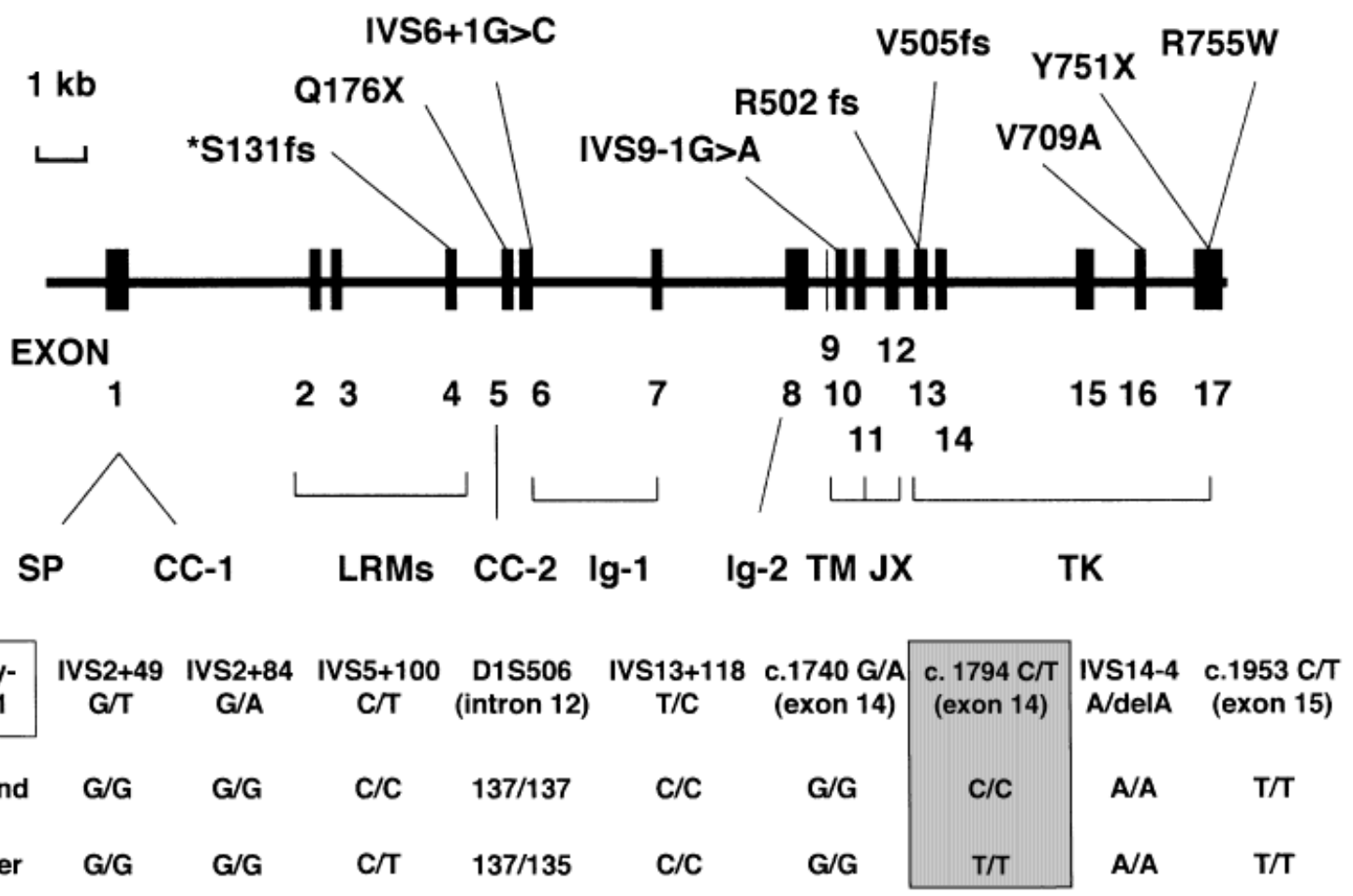

FIGURE 1. Disease-associated mutations in the TRKA and polymorphism analysis in family KI-111. Mutations reported in this study are listed above the TRKA gene diagram. Asterisk indicates mutation previously reported [Miura et al., $2000 \mathrm{~b}$ ]. Abbreviations listed below show the domain structures encoded by the corresponding exon(s) [Indo et al., 1997; Schneider and Schweiger, 1991]. SP = signal peptide; CC-1 and CC-2 = the first and second cysteine clusters, respectively; LRMs = leucine-rich motifs; Ig-1 and Ig-2 = the first and second immunoglobulin-like motifs, respectively; TM = transmembrane; $\mathrm{JX}=$ juxtamembrane; TK = tyrosine kinase. Amino acid numbering of the TRKA protein and structure of the TRKA gene are described, according to Martin-Zanca et al. [1989] and Indo et al. [1997], respectively. A nomenclature system for human gene mutations is used, according to the recommendations [Antonarakis, 1998]. Polymorphism analysis of the TRKA locus, including eight single nucleotide polymorphisms (SNPs) and a microsatellite (D1S506), is shown for Family KI-111 in lower part. A shaded box indicates the SNP locus that shows the non-Mendelian inheritance of the TRKA in this family.

pound heterozygote, with two different mutations at the TRKA locus on separate chromosomes (Table 1 and Fig. 1). One contained a $\mathrm{C}$ to G transversion at nucleotide 2337 (c.2337C > G) in exon 17, which changes Tyr to a termination codon at amino acid 751 (Tyr751Ter). The other was a $\mathrm{C}$ to $\mathrm{T}$ transition at nucleotide 2347 (c.2347C>T) in exon 17, which causes an Arg to Trp substitution at amino acid 755 (Arg755Trp). DNA samples from both parents were not available for sequence analysis. We examined nine polymorphic sites in the TRKA locus and determined haplotypes based on combination of polymorphism on these sites. We have found that the patient is heterozygous for two sites (D1S506 and c.1740G/A), indicating that she has two alleles (data not shown). One mutant allele (Arg755Trp) in this patient is probably linked to the same haplotype as that detected in the patient KI-116 who has ho- mozygous for Arg755Trp mutation, as described below. Thus, analysis of polymorphic sites in the TRKA locus showed that the patient has two haplotypes, suggesting that two TRKA mutations in exon 17 are located on two separate chromosomes.

In a male CIPA patient of family KI-115, sequencing of genomic DNA revealed that a 3' splice site of intron 9 contained a $\mathrm{G}$ to $\mathrm{A}$ transition at the first position (IVS9-1G>A) (Table 1 and Fig. 1). Mother was also sequenced. The proband and his mother were homozygous and heterozygous for IVS9-1G>A, respectively. DNA sample from his father was not available for testing. We determined the haplotypes of the proband in family KI-115. The proband was homozygous for nine polymorphic sites of the TRKA locus. This strongly suggests that parents share the same TRKA mutant allele linked to the same haplotype, although they denied con- 
sanguinity. Dr. E. Beutler's laboratory did an analysis of the pyruvate kinase gene (PKLR) and detected a $G$ to $A$ transition at nucleotide 1378 that causes a Val to Met substitution at amino acid 460 (Val460Met) (data not shown). The proband and both parents were homozygous and heterozygous for the mutation, respectively. This mutation has been previously found in PK deficient patients [Baronciani and Beutler, 1995]. Thus, this Hispanic patient has two autosomal genetic disorders, CIPA and pyruvate kinase deficiency, whose genetic loci are both mapped to $1 \mathrm{q} 21-22$.

In family KI-116, a homozygous $\mathrm{C}$ to $\mathrm{T}$ transition at nucleotide 2347 (c.2347C > T) in exon 17 was predicted to cause an Arg to Trp substitution at amino acid 755 (Arg755Trp) (Table 1 and Fig. 1). This substitution was the same as one allele detected in family KI-114. Parents were also sequenced. The proband and both parents were homozygous and heterozygous for the substitution, respectively. We determined the haplotypes of the proband in family KI-116. The proband was homozygous for nine polymorphic sites of the TRKA locus. This strongly suggests that parents share the same TRKA mutant allele linked to the same haplotype, although they denied consanguinity. Interestingly, Arg755Trp mutation was linked to one of two haplotypes observed in the proband of family KI-114.

In family KI-25, the female patient was a compound heterozygote, with two different mutations at the TRKA locus, on separate chromosomes (Table 1 and Fig. 1). In one allele, a 5' splice site of intron 6 contained a $\mathrm{G}$ to $\mathrm{C}$ transversion in the first position (IVS6 $+1 \mathrm{G}>\mathrm{C}$ ) (Table 1 and Fig. 1). The other was a $T$ to $C$ transition at nucleotide 2210 (c.2210T $>$ C) in exon 16, which causes a Val to Ala substitution at amino acid 709 (Val709Ala). Mother was also sequenced. The latter mutation was derived from her mother. DNA sample from the father was not available for testing.

\section{Allelotype Analysis of the Proband and Mother in Family KI-111}

To determine whether this non-Mendelian inheritance in family KI-111 involved all or part of chromosome 1, we did an allelotype analysis for the patient and her mother. The patient was homozygous at the D1S506 and its three neigh- boring microsatellites (D1S498, D1S484, and D1S2878). There was heterozygosity in other microsatellite loci on chromosome 1 and she shared at least one allele with her mother in each locus (Fig. 2).

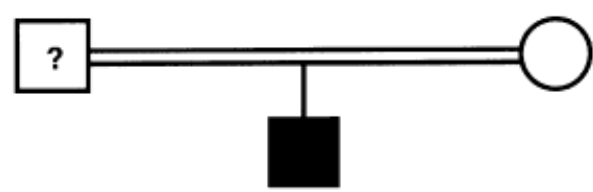

\begin{tabular}{|c|c|c|c|c|}
\hline D1S214 & 138 & 122 & 138 & 122 \\
\hline D1S450 & 338 & 342 & 344 & 342 \\
\hline D1S2667 & 136 & 138 & 146 & 138 \\
\hline D1S2697 & 299 & 291 & 291 & 291 \\
\hline D1S234 & 273 & 269 & 275 & 269 \\
\hline D1S255 & 88 & 94 & 94 & 94 \\
\hline D1S2797 & 116 & 126 & 114 & 126 \\
\hline D1S2890 & 224 & 224 & 222 & 224 \\
\hline D1S230 & 149 & 149 & 149 & 149 \\
\hline D1S2841 & 234 & 234 & 238 & 234 \\
\hline D1S207 & 160 & 160 & 160 & 160 \\
\hline D1S206 & 210 & 214 & 210 & 214 \\
\hline D1S2726 & 286 & 286 & 286 & 286 \\
\hline D1S252 & 97 & 95 & 97 & 95 \\
\hline D1S498 & 203 & 203 & 203 & 203 \\
\hline D1S506 & 137 & 137 & 135 & 137 \\
\hline D1S484 & 276 & 276 & 276 & 276 \\
\hline D1S2878 & 168 & 168 & 172 & 168 \\
\hline D1S196 & 333 & 321 & 331 & 321 \\
\hline D1S218 & 277 & 279 & 269 & 279 \\
\hline D1S238 & 310 & 310 & 310 & 310 \\
\hline D1S413 & 250 & 254 & 254 & 254 \\
\hline D1S249 & 177 & 167 & 177 & 167 \\
\hline D1S425 & 348 & 346 & 348 & 346 \\
\hline D1S213 & 126 & 108 & 116 & 108 \\
\hline D1S2800 & 209 & 217 & 217 & 217 \\
\hline D1S2785 & 182 & 180 & 171 & 180 \\
\hline D1S2842 & 352 & 348 & 352 & 348 \\
\hline D1S2836 & 251 & 243 & 243 & 243 \\
\hline
\end{tabular}

FIGURE 2. Allelotype analysis of the proband and her mother with chromosome 1 markers in family KI-111. The polymorphic markers used for this analysis are shown on the left. Twenty-nine polymorphic markers were located on chromosome 1 from the $1 \mathrm{p}$ to $1 \mathrm{q}$ terminal regions. Alleles are labeled arbitrarily, according to size. The allelotypes of the proband and her mother are shown with one possible phasing. The father's DNA was not available for analysis. An arrow indicates the locus of the TRKA. The PKLR gene is located between microsatellites D1S498 and D1S484. Shaded numbers (proband) show alleles not detected in the mother and are probably derived from the father. A shaded box indicates the genomic region where a putative recombination event occurred during paternal gametogenesis. We assume that father and mother share one chromosome 1 not carrying the TRKA mutation, probably due to consanguinity. 


\section{DISCUSSION}

The human TRKA gene on chromosome 1q21-q22, estimated to span $>23 \mathrm{~kb}$ is divided into 17 exons and 16 introns [Indo et al., 1997; Weier et al., 1995], coding the receptor tyrosine kinase of 790 or 796 amino acid residues (Fig. 1). A single transmembrane domain divides the TRKA protein into an extracellular and an intracellular domain [Barbacid, 1995; Bothwell, 1995; Schneider and Schweiger, 1991]. The extracellular domain, important for specific NGF binding, includes a signal peptide, three tandem leucine-rich motifs flanked by two cysteine clusters, and two immunoglobulin-like domains (or motifs). The intracellular domain includes a juxtamembrane region, a tyrosine kinase domain, and a very short carboxyl-terminal tail. There is a general correlation between the genomic organization of TRKA and the functional organization of the TRKA protein product [Indo et al., 1997].

In this report, we analyzed all coding exons from genomic DNA by PCR direct sequencing. A total of eight novel mutations were detected in nine families from five countries. CIPA mutations are spread throughout extracellular and intracellular domains. These are two missense mutations (Val709Ala and Arg755Trp), two frameshift mutations (Arg502fs and Val505fs), two nonsense mutations (Gln176Ter and Tyr751Ter), and two splice mutations (IVS6 + $1 \mathrm{G}>\mathrm{C}$ and IVS9-1G $>\mathrm{A}$ ).

Two missense mutations, Val709Ala and Arg755Trp, are probably responsible for CIPA. They have not been observed in 100 normal chromosomes. The Val709Ala mutation is located in the intracellular tyrosine kinase domain and conserved among receptor tyrosine kinases, including human TRKB and TRKC [MartinZanca et al., 1989; Nakagawara et al., 1995], which suggests that it is important for enzyme activity. We previously reported that the preceding residue (Gly708Ser) is also is mutated in CIPA [Mardy et al., 1999]. The latter mutant was expressed and its product was processed as the wild type TRKA, but it showed a significantly diminished NGF-stimulated autophosphorylation of the TRKA protein [Mardy et al., 2001]. It is likely that Val709Ala mutation also affects autophosphorylation of the TRKA protein. Arg755Trp mutation, located in the intracellular tyrosine kinase domain, was detected in a state of either compound heterozygosity or ho- mozygosity, in two patients. The compound heterozygous patient from family KI-114 has a nonsense mutation (Tyr751Ter) on a separate chromosome. Parents of the homozygous patient from family KI-116 are heterozygous for the Arg755Trp mutation. A consensus sequence motif YXXM, which interacts phosphatidylinositol 3-kinase, is located at the end of tyrosine kinase catalytic domain of the TRKA protein (Tyr-751 in TRKA protein) [Barbacid, 1995]. A bulky Trp substitution at the succeeding residue $(-755)$ of the motif may affect the function of kinase catalytic domain. These data strongly suggest that $A r g 755$ Trp mutation is responsible for CIPA in the two families. Two European families KI-114 and KI116 probably share Arg755Trp mutation linked to the same haplotype in the TRKA locus. It is likely that Arg755Trp mutation is derived from the same ancestry. Effects of these two missense mutations on the function of the TRKA protein are currently unknown, and experiments are in progress to address this question.

In the present study, the frameshift mutation, Ser131fs, was detected as a homozygous state in an Italian patient. Ser131fs is located in the third leucine-rich motif of the extracellular domain [Indo et al., 1997; Mardy et al., 1999]. This should result in truncation of the TRKA polypeptide in the extracellular domain. Thus, the mutation almost certainly causes defect in NGF signal transduction. Interestingly, the same mutation was previously reported as one allele of a Japanese patient who was a compound heterozygote for the TRKA locus [Miura et al., 2000b]. Haplotype analysis of the TRKA locus in these two patients suggested that the frameshift mutation occurred independently (data not shown). The mutation involves a deletion of two bases TC in repeat sequence (TC) ${ }_{4}$ encompassing nucleotides 469-476. We speculate the slipped strand mispairing as a common mechanism for the mutation detected in two separate countries [Levinson and Gutman, 1987].

Both Arg502fs andVal505fs are located in the junction between juxtamembrane and tyrosine kinase domains. These should result in truncation of the TRKA polypeptide. Thus, these mutations almost certainly cause defects in NGF signal transduction.

The two nonsense mutations, Gln176Ter and Tyr751Ter, are located on the second cysteine 
cluster of the extracellular domain and the intracellular tyrosine kinase domain, respectively. Gln176Ter should result in truncation of the TRKA polypeptide in the extracellular domain, causing defect in the function of this receptor tyrosine kinase for NGF. Tyr751Ter mutation is interesting since it substitutes the Tyr-751 residue in the consensus sequences motif YXXM, which interacts phosphatidylinositol 3'-kinase and is located at the end of the kinase catalytic domain [Barbacid, 1995]. This should result in truncation of the TRKA protein at the end of the kinase catalytic domain. The truncated TRKA protein also lacks the Tyr-785 residue which is located in the short carboxy terminal tail and is important for binding of phospholipase C $\gamma$ [Barbacid, 1995]. Thus, Tyr751Ter probably causes defect in NGF signal transduction.

Effects of splice mutations are difficult to demonstrate in vivo because CIPA patients lack neurons that express the TRKA gene. Two splice site mutations (IVS6 $+1 \mathrm{G}>\mathrm{C}$ and IVS9-1G $>\mathrm{A}$ ) are predicted to cause skipping of exons 6 and 10, respectively. These changes would result in deletions of a part of the first immunoglobulin-like domain and a part of the transmembrane domain, respectively. The first immunoglobulinlike domain of the TRKA protein contains two conserved cysteines presumably involved in generating the canonical disulfide bond [Barbacid, 1995]. The domain is encoded by two exons, 6 and 7 , each containing one cysteine codon [Indo et al., 1997]. This splicing mutation is predicted to cause deletion of a part of the first immunoglobulin-like domain as well as disruption of the canonical disulfide bond. Thus, IVS6 $+1 \mathrm{G}>\mathrm{C}$ is likely to affect the binding of NGF to the extracellular domain. Exon 10 of the TRKA encodes a part of transmembrane domain that probably participates in joining the extracellular domain and intracellular domain [Indo et al., 1997]. TRKA receptor becomes activated by NGF-mediated dimerization of receptor molecules at the cell surface followed by autophosphorylation of their intracellular tyrosine residues [Barbacid, 1995]. Thus, the splice mutation IVS9-1G $>$ A is also likely to cause a defect in NGF signal transduction.

Non-Mendelian inheritance of the TRKA mutation in family KI-111 might be due to paternal uniparental disomy. Uniparental disomy is defined as the presence of a chromosome pair that derives from only one parent in a diploid individual [Engel, 1980]. Heterodisomy is the inheritance of a pair of chromosome homologs from a parent, whereas isodisomy implies the inheritance of two copies of a single parental homolog. We reported complete paternal uniparental isodisomy for chromosome 1 , as revealed by mutation analyses of the TRKA gene in a patient with CIPA [Miura et al., 2000a]. But a different mechanism must be considered here since allelotype analysis revealed the presence of two chromosomes in the patient in family KI-111. We speculate a similar mechanism to that described for a patient with Herlitz junctional epidermolysis bullosa in whom maternal uniparental disomy of chromosome 1 with reduction to homozygosity of the LAMB3 locus was revealed [Pulkkinen et al., 1997]. The CIPA phenotype in family KI-111 might be due to paternal uniparental primary heterodisomy and meroisodisomy of a region encompassing the TRKA locus on chromosome 1q, resulting in reduction to homozygosity of the paternally derived insertion mutation within the TRKA gene. Recombination event(s) probably happened during paternal gametogenesis in a region encompassing the TRKA gene. A putative mechanism for this chromosomal aberration could be nondisjunction at the first meiotic division, preceded by recombination events and followed by either gamete complementation at fertilization or trisomy rescue at postzygotic mitosis. The patient shares at least one allele with her mother in all microsatellite loci studied on chromosome 1. This could be attributed to consanguinity of her parents. We could not ascertain this hypothesis because the DNA sample from her father was not available for testing. Of course one cannot completely exclude another mechanism(s) for CIPA in this family.

In this report, we have described a patient with two autosomal recessive disorders, CIPA and pyruvate kinase deficiency, whose genetic loci are both mapped to a closely linked region on 1q21-q22 [Satoh et al., 1988]. Both are located between D1S498 and D1S484 (Fig. 2: left). Dr. E. Beutler's laboratory and our study each detected a homozygous missense mutation in the PKLR gene and a homozygous splice mutation in the TRKA gene, respectively. Thus, concomitant occurrence of two disorders in this family is ascribed to a combination of two separate mu- 
tant genes, but not to a contiguous gene syndrome. In addition, there were no unusual clinical findings in a Hispanic patient with the same homozygous PKLR mutation previously reported [Baronciani and Beutler, 1995], suggesting that the linkage of these two disorders seems to be limited to the present family. Generally, concomitant occurrence of two or more genetic disorders mapped to the same chromosome region suggests the possibility of a contiguous gene syndrome. But the present case indicates that one cannot rule out a combination of two separate mutant genes. This might be a rare but novel mechanism that is responsible for two autosomal genetic disorders in a patient.

Mutations responsible for CIPA are located in the extracellular domain as well as in the intracellular domain of TRKA [Mardy et al., 1999; Miura et al., 2000b]. Mutations in the TRKA gene are heterogeneous, although relatively common founder mutations occurred in some ethnic groups [Miura et al., 2000b; Shatzky et al., 2000]. These data further confirm findings that TRKA defects cause CIPA in various ethnic groups. CIPA is a painless but severe genetic disorder associated with devastating complications, often leading to crippling or fatal consequences. This study will facilitate analyses of CIPA mutations in TRKA and aid in diagnosing this disorder and for genetic counseling.

\section{ACKNOWLEDGMENTS}

We are grateful to all the CIPA patients and their family members for their kind cooperation. We thank Dr. H. Yamada and M. Inutsuka for providing samples. We also thank for Dr. Ernest Beutler at the Scripps Research Institute for mutation analysis of the PKLR gene in family KI115 and for clinical information on patients with the PKLR mutation that they described previously. M. Ohara provided language assistance.

\section{REFERENCES}

Antonarakis SE, Nomenclature Working Group. 1998. Recommendations for a nomenclature system for human gene mutations. Hum Mutat 11:1-3.

Barbacid M. 1995. Structural and functional properties of the TRK family of neurotrophin receptors. Ann NY Acad Sci 766:442-458.

Baronciani L, Beutler E. 1995. Molecular study of pyruvate kinase deficient patients with hereditary nonspherocytic hemolytic anemia. J Clin Invest 95:1702-1709.
Bothwell M. 1995. Functional interactions of neurotrophins and neurotrophin receptors. Ann Rev Neurosci 18:223-253.

Cargill M, Altshuler D, Ireland J, Sklar P, Ardlie K, Patil N, Shaw N, Lane CR, Lim EP, Kalyanaraman N, Nemesh J, Ziaugra L, Friedland L, Rolfe A, Warrington J, Lipshutz R, Daley GQ, Lander ES. 1999. Characterization of singlenucleotide polymorphisms in coding regions of human genes. Nat Genet 22:231-238.

Dyck PJ. 1984. Neuronal atrophy and degeneration predominantly affecting sensory and autonomic neurons. In: Dyck PJ, Thomas PK, Lambert EH, Bunge R, editors. Peripheral neuropathy. Philadelphia: W.B. Saunders Company. p 1557-1599.

Engel E. 1980. A new genetic concept: uniparental disomy and its potential effect, isodisomy. Am J Med Genet 6:137-143.

Greco A, Villa R, Tubino B, Romano L, Penso D, Pierotti MA. 1999. A novel NTRK1 mutation associated with congenital insensitivity to pain with anhidrosis. Am J Hum Genet 64:1207-1210.

Indo Y, Tsuruta M, Hayashida Y, Karim MA, Ohta K, Kawano T, Mitsubuchi H, Tonoki H, Awaya Y, Matsuda I. 1996. Mutations in the TRKA/NGF receptor gene in patients with congenital insensitivity to pain with anhidrosis. Nat Genet 13:485-488.

Indo Y, Mardy S, Tsuruta M, Karim MA, Matsuda I. 1997. Structure and organization of the human TRKA gene encoding a high affinity receptor for nerve growth factor. Jpn J Hum Genet 42:343-351.

Kaplan DR, Hempstead BL, Martin-Zanca D, Chao MV, Parada LF. 1991. The trk proto-oncogene product: a signal transducing receptor for nerve growth factor. Science 252:554-558.

Klein R, Jing S, Nanduri V, O'Rourke E, Barbacid M. 1991. The trk proto-oncogene encodes a receptor for nerve growth factor. Cell 65:189-197.

Levi-Montalcini R. 1987. The nerve growth factor: thirtyfive years later. EMBO J 6:1145-1154.

Levinson G, Gutman GA. 1987. Slipped-strand mispairing: a major mechanism for DNA sequence evolution. Mol Biol Evol 4:203-221.

Mardy S, Miura Y, Endo F, Matsuda I, Sztriha L, Frossard P, Moosa A, Ismail EAR, Macaya A, Andria G, Toscano E, Gibson W, Graham GE, Indo Y. 1999. Congenital insensitivity to pain with anhidrosis: novel mutations in the TRKA (NTRK1) gene encoding a high-affinity receptor for nerve growth factor. Am J Hum Genet 64:1570-1579.

Mardy S, Miura Y, Endo F, Matsuda I, Indo Y. 2001. Congenital insensitivity to pain with anhidrosis (CIPA): effect of TRKA (NTRK1) missense mutations on autophosphorylation of the receptor tyrosine kinase for nerve growth factor. Hum Mol Genet 10:179-188.

Martin-Zanca D, Oskam R, Mitra G, Copeland T, Barbacid M. 1989. Molecular and biochemical characterization of the human trk proto-oncogene. Mol Cell Biol 9:24-33.

McKusick VA. 1994. Mendelian inheritance in man. Baltimore: The Johns Hopkins University Press. p 2073. 
Miura Y, Hiura M, Torigoe K, Numata O, Kuwahara A, Matsunaga M, Hasegawa S, Boku N, Ino H, Mardy S, Endo F, Matsuda I, Indo Y. 2000a. Complete paternal uniparental isodisomy for chromosome 1 revealed by mutation analyses of the TRKA (NTRK1) gene encoding a receptor tyrosine kinase for nerve growth factor in a patient with congenital insensitivity to pain with anhidrosis. Hum Genet 107:205-209.

Miura Y, Mardy S, Awaya Y, Nihei K, Endo F, Matsuda I, Indo Y. 2000b. Mutation and polymorphism analysis of the TRKA (NTRK1) gene encoding a high-affinity receptor for nerve growth factor in congenital insensitivity to pain with anhidrosis (CIPA) families. Hum Genet 106:116-124.

Nakagawara A, Liu XG, Ikegaki N, White PS, Yamashiro DJ, Nycum LM, Biegel JA, Brodeur GM. 1995. Cloning and chromosomal localization of the human TRK-B tyrosine kinase receptor gene (NTRK2). Genomics 25:538-546.

Pavone L, Huttenlocher P, Siciliano L, Micali G, Rizzo R, Anastasi M, Maimone D, Woolmann R. 1992. Two brothers with a variant of hereditary sensory neuropathy. Neuropediatrics 23:92-95.

Pulkkinen L, Bullrich F, Czarnecki P, Weiss L, Uitto J. 1997. Maternal uniparental disomy of chromosome 1 with reduction to homozygosity of the LAMB3 locus in a patient with Herlitz junctional epidermolysis bullosa. Am J Hum Genet 61:611-619.

Satoh H, Tani K, Yoshida MC, Sasaki M, Miwa S, Fujii H.
1988. The human liver-type pyruvate kinase (PKL) gene is on chromosome 1 at band q21. Cytogenet Cell Genet $47: 132-133$.

Schneider R, Schweiger M. 1991. A novel modular mosaic of cell adhesion motifs in the extracellular domains of the neurogenic trk and trkB tyrosine kinase receptors. Oncogene 6:1807-1811.

Shatzky S, Moses S, Levy J, Pinsk V, Hershkovitz E, Herzog L, Shorer Z, Luder A, Parvari R. 2000. Congenital insensitivity to pain with anhidrosis (CIPA) in Israeli-Bedouins: genetic heterogeneity, novel mutations in the TRKA/NGF receptor gene, clinical findings, and results of nerve conduction studies. Am J Med Genet 92:353-360.

Swanson AG. 1963. Congenital insensitivity to pain with anhidrosis. Arch Neurol 8:299-306.

Thoenen H, Barde YA. 1980. Physiology of nerve growth factor. Physiol Rev 60:1284-1335.

Weier HUG, Rhein AP, Shadravan F, Collins C, Polikoff D. 1995. Rapid physical mapping of the human trk protooncogene (NTRK1) to human chromosome 1q21-q22 by P1 clone selection, fluorescence in situ hybridization (FISH), and computer-assisted microscopy. Genomics 26:390-393.

Yotsumoto S, Setoyama M, Hozumi H, Mizoguchi S, Fukumaru S, Kobayashi K, Saheki T, Kanzaki T. 1999. A novel point mutation affecting the tyrosine kinase domain of the TRKA gene in a family with congenital insensitivity to pain with anhidrosis. J Invest Dermatol 112:810-814. 